\title{
Research on method for high sensitive detection of harmful gases in livestock houses based on laser absorption spectrum
}

\author{
Runyu Wang ${ }^{1}$, Daming Dong ${ }^{1,2}$, Zengtao $\mathrm{Ji}^{3}$, Leizi Jiao ${ }^{1,2^{*}}$ \\ ${ }^{1}$ School of Electronic Engineering and Automation, China Guangxi Key Laboratory of Optoelectronic Information Processing, Guilin \\ University of Electronic Technology, Guilin 541004, China \\ ${ }^{2}$ Beijing Research Center of Intelligent Equipment for Agriculture, Beijing Academy of Agriculture and Forestry Sciences, Beijing \\ 100097, China \\ ${ }^{3}$ Beijing Research Center for Information Technology in Agriculture, Beijing Academy of Agriculture and Forestry Sciences, Beijing \\ 100097, China
}

\begin{abstract}
Harmful gases such as ammonia and hydrogen sulfide in livestock and poultry houses can seriously damage the health of livestock and poultry as well as animal keepers, so it is great significant to detect these harmful gases rapidly and accurately for the improvement of the welfare of animals and the health of animal keepers. Laser absorption spectroscopy is a gas detection method with the advantages of high sensitivity and selectivity, and is widely used in industrial gas detection. However, it needs further exploring to verify whether laser absorption spectroscopy is useful in detecting low concentration harmful gases in livestock and poultry houses. This paper researches on the method for high-sensitivity detection of harmful gases in livestock and poultry houses based on laser absorption spectroscopy by detecting the absorption signals of ammonia with a self-designed system including a tunable laser wavelength scanning system, a photoelectric detecting system and a long light path gas absorption well, and verifies that laser absorption spectroscopy can be used for detecting harmful gases in livestock and poultry houses.
\end{abstract}

\section{Introduction}

With the development of economy, livestock breeding has shifted from retail breeding to large-scale breeding. By using large-scale breeding approach, many harmful gases such as ammonia (NH3), hydrogen sulfide (H2S) and methane $(\mathrm{CH} 4)$ will be produced while the animals breathe, waste breaks down and padding and facilities decompose. Especially in cold winter, the livestock and poultry houses are often closed to keep a suitable temperature for the growth of the animals and cannot be ventilated regularly, and thus the concentration of NH3, $\mathrm{H} 2 \mathrm{~S}, \mathrm{CH} 4$ and other harmful gases remains at a high level, which will make the animals sick or even dead[1]. Those harmful gases in the livestock and poultry houses will pollute the environment when released into the air, creating hazardous air quality and exacerbating the living environment of human beings. What's more, it may harm the health of workers and surrounding residents, causing respiratory diseases[2]. Therefore, rapid and accurate detection of harmful gases, timely ventilation, disinfection and deodorization are not only the health needs of animals and people, but also urgent demands of environment protection and food safety.

Electrochemical sensors are small in size, cost effective and easy to operate in gas detection, but currently, they are mainly applied in industrial field because their detection range and sensibility cannot meet the requirements of harmful gas detection in livestock and poultry houses. More importantly, electrochemical sensors have the disadvantages of low stability, serious linear drift, cross absorption and short life span in a complicated agricultural environment[3], thus they are not suitable for stable and accurate harmful gas detection in livestock and poultry houses for long time. That is the main reason why electrochemical sensors are not widely applied in gas detection in livestock and poultry houses.

Tunable diode laser absorption spectroscopy (TDLAS)[4] takes advantage of the properties of tunable wavelength and narrow linewidth of semiconductor diode laser and is used for measuring the concentration of gases rapidly by selecting a suitable absorption line of the to-be-detected gases and removing the interference of other gases[5]. Compared with other complex systematic analysis methods, TDLAS has the advantages of high sensitivity, high selectivity, rapid response[6], small size and easy operation, and thus it is widely used in industrial gas detection.[7] However, it still needs further studying and verifying to see whether TDLAS can be used for detecting harmful gases stably and accurately in complicated livestock and poultry houses for long time. This paper researches on the method for high-sensitivity detection of harmful gases in livestock and poultry houses based on laser absorption spectroscopy by detecting the ammonia with a TDLAS system, and provides methods and technical supports for intelligent gas monitoring system in livestock and poultry houses. 


\section{System Design}

The key points of TDLAS high sensitive gas detection are accurate scanning of laser wavelength, accurate extraction of harmonic signals and increase of light path of absorption well. The TDLAS gas detection system mainly consists of a laser wave scanning part, a harmonic signal extracting part and a long path gas absorption cell. The overall scheme and principle is shown in Fig. 1.

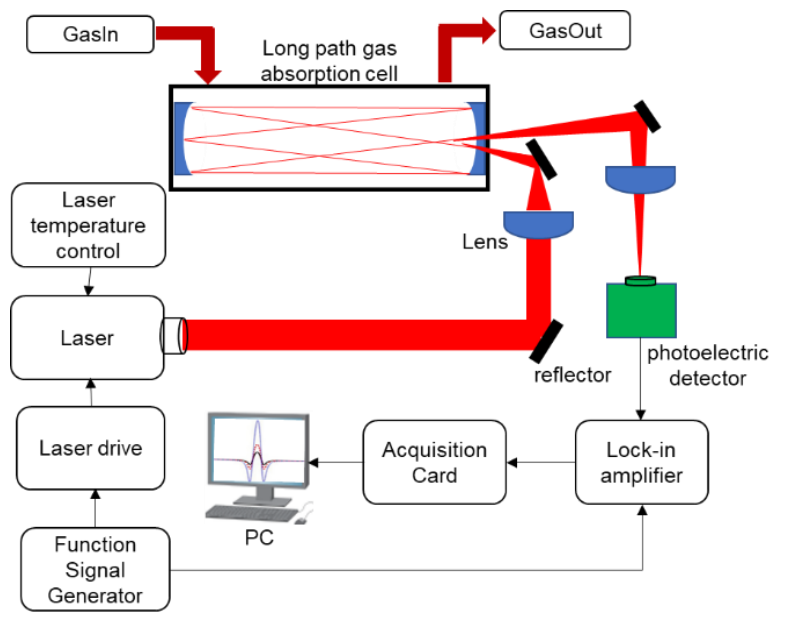

Fig. 1. Principle diagram of high-sensitivity detection of harmful gases in livestock based on TDLAS

\subsection{Wavelength scanning system of tunable laser}

A tunable quantum well DFB laser (Wuhan Six-Nine Technology Ltd., Wuhan, China) is used in the system. The central wavelength is $1512 \mathrm{~nm}$, the power is $5 \mathrm{~mW}$, the spectral bandwidth is $0.2 \mathrm{~nm}$, current drift coefficient is $0.01 \mathrm{~nm} / \mathrm{mA}$, and temperature drift coefficient is 0.1 $\mathrm{nm} /{ }^{\circ} \mathrm{C}$. A butterfly pigtail package is used. A semiconductor refrigerating unit is built inside, which enables the laser works at a stable power and keeps wavelength stable through high precision temperature control. Laser wavelength scanning is operated by changing the current of DFB laser under constant temperature. WLD3343 (Wavelength Electronics) is a high precision laser driver chip, which can supply up to $500 \mathrm{~mA}$ precision current to the laser from a single $5 \mathrm{~V}$ power source without any radiating unit. The current of the chip can be controlled and monitored by a voltage signal, so that the laser can be protected from static electricity. WTC3243 (Wavelength company, city, country) is a compact analog PI control loop circuit optimized for use in ultrastable temperature control applications with a stability of better than $0.0009^{\circ} \mathrm{C}$. The temperature is set up with a voltage signal, so a high precision linear regulator is needed to supply precision stable voltage, so as to control the temperature accurately and precisely. The circuit diagram of the laser wavelength scanning system based on the laser, temperature control and driver chip is shown in Fig. 2. The system uses a $5 \mathrm{~V}$ power supply. An AD780 ultrahigh precision band gap reference voltage is used to provide a $2.5 \mathrm{~V}$ output for setting up and controlling the temperature of the laser, and the voltage of the laser can be shifted from 0 to $2.5 \mathrm{~V}$ with a high precision slide rheostat, so that the temperature of the laser can be changed. In this system, the voltage is set up as $1 \mathrm{~V}$, and the temperature of the laser is $25^{\circ} \mathrm{C}$. Laser current driven signal interfaces are reserved on the circuit board of the system, so that different signal-driven lasers can be connected on the board conveniently. The system uses PC104 standard interfaces, and can be connected with HE104 power board conveniently. (Fig. 2).

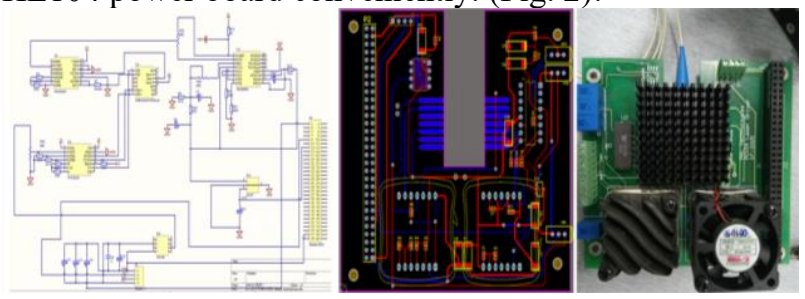

Fig. 2 Laser wavelength scanning system

\subsection{Photoelectric conversion circuit design}

A photoelectric detection circuit is crucial for laser signal detection, since its performance can affect the direct absorption and detection of harmonic signals directly. The photoelectric detection circuit is mainly composed of a photoelectric detector and a photoelectric conversion circuit, wherein the photoelectric detector is used for receiving laser signals and converting them into current signals. An indium potassium arsenic photodiode is used as the photoelectric detector, which has the features of low temperature drift, low dark noise, low junction capacitance and high response. G10899-01K (Hamamatsu company) is characterized by wide spectral response range, low dark current and low junction capacitance, and thus is suitable for detecting weak absorption optical signals. A typical trans-impedance amplifier[8] is chosen as the pre-amplification electric circuit, and an LM6172 (Texas Instruments) amplifier chip is used. The working principle is selecting the compensating capacitance value according to the junction capacitance and equivalent input capacitance of the photoelectric detector and amplifier chip, and converting the current signal to voltage signal and amplifying the voltage signal. The pre-amplification electric circuit is shown in Fig. 3. The output signal enters into SR830 lock-in amplifier (Stanford) and then the harmonic signal is extracted.
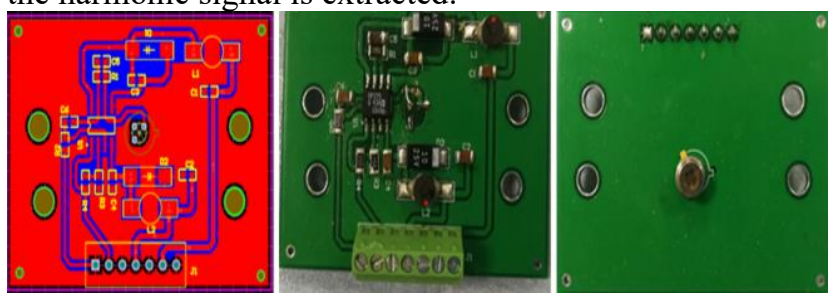

Fig. 3 photoelectric converting system

\subsection{Design of long path gas absorption cell}

According to Lambert Beer's law[9], the concentration of the gas is proportional to the path length of the light 
through the gas in gas detection based on laser absorption spectroscopy. Therefore, it is crucial to increase the path length of the light through the gas in high sensitive gas detection. In this system, the gas absorption well is a White cell[10], which is constructed using a multiple of mirrors to realize many times of reflection and therefore increase the light path of the absorption well. There are 48 traversals (Fig. 4), the reflective distance of each traversal is $0.09 \mathrm{~m}$, and the valid light path can be as long as about $4.3 \mathrm{~m}$. However, the quality of the laser signal will be affected by the reflectivity and smoothness of the reflection mirrors after the light is reflected for many times. To ensure the transmission quality of the laser beam after multiple reflections, the reflection mirrors are designed in concave surface and with gold-plated film. In this way, the reflectivity of the mirrors can be very high, the diameter of the laser beam is small, thus the wastage of laser power is reduced and the laser beam received by the photoelectric detector is improved.

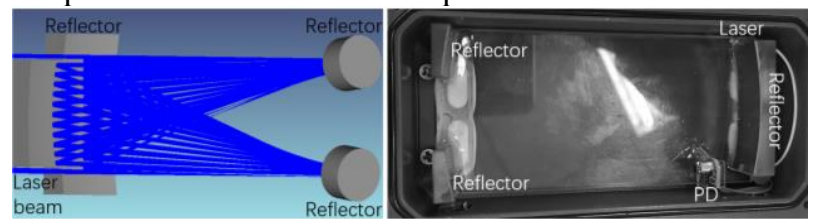

Fig. 4 Diagram of long path gas absorption well

\section{Results and Analysis}

The real equipment photo of ammonia detection (5 ppm) based on TDLAS is shown in Fig. 5. While detecting, the concentration of the ammonia can be tuned over with a gas control valve. A low-frequency scanning sawtooth signal $(5 \mathrm{~Hz})$ and a high-frequency modulated sinusoidal signal $(5 \mathrm{KHz})$ are input through the current control interface of the WTC3243 laser driver and work as the laser driver signal (Fig. 5). The direct absorption signal caused by gas absorption and harmonic absorption signal are recorded by oscilloscope and self-developed testing software at the same time (Fig. 5). It proves that TDLAS system cannot observe the direct absorption signal when ammonia enters into the gas absorption well through extraction pipe, but it can observe the harmonic absorption signal, proving that the system is able to detect ammonia and that harmonic absorption signal is more sensitive compared with direct absorption signal.

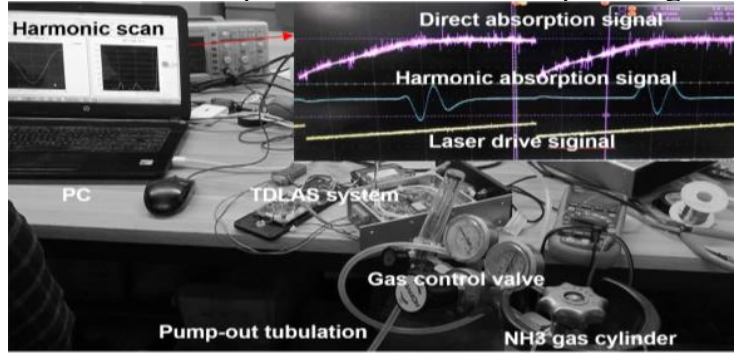

Fig. 5 Real equipment photo of ammonia detection based on TDLAS with long light path

\section{Conclusion}

In this paper, a high precision laser wavelength scanning system, a photoelectric conversion system and a long light path gas absorption well are designed based on the principle of tunable diode laser absorption spectroscopy. The detection results show that TDLAS system can be used for detecting harmonic signals created by ammonia with a concentration of lower than $5 \mathrm{ppm}$, and can be used for high-sensitivity detection of low concentration ammonia and other harmful gases in livestock and poultry houses. This system also provides theoretical basis and technical support for intelligent air quality detection and control in livestock and poultry houses.

\section{Acknowledgement}

The authors gratefully acknowledge support from the Innovation Capacity Building Project of Beijing Academy of Agriculture and Forestry Sciences (KJCX20200417), Major Scientific and Technological Innovation Project (MSTIP): the Research and Demonstration on Key Technologies of Precision Breeding and Management of Laying Hens (2019JZZY020611), and Guangxi Key Laboratory of Optoelectroric Information Processing(Guilin University of Electronic Technology) (GD18204).

\section{References}

1. Chunmei Li, Mingyang Li, Dan Shen, Yansen Li, Guofeng Han. Research Progress of the effects of environmental key factors on animal health in livestock houses[J]. China Poultry, 2020, 42(3): 001

2. Huan Wang, Yu Zhang, Qi Han, Yanmin Xu, Guanghui Hua, Houjuan Xing. The inflammatory injury of heart caused by ammonia is realized by oxidative stress and abnormal energy metabolism activating inflammatory pathway[J]. Science of The Total Environment, 2020,742: 140532.

3. Lekha S, Suchetha M. Recent advancements and future prospects on E-Nose sensors technology and machine learning approaches for non-invasive diabetes diagnosis: A Review[J]. IEEE Reviews in Biomedical Engineering, 2020: 2993591.

4. Schiff H I, Mackay G I, Bechara J. The use of tunable diode laser absorption spectroscopy for atmospheric measurements[J]. Research on Chemical Intermediates, 1994, 20(3-5): 525-556.

5. Tang C L. Laser modulation spectroscopy of solids[J]. Journal of Applied Physics, 1974, 45(10): 4503.

6. Schilt S, Thevenaz L, Robert P. Wavelength modulation spectroscopy: Combined frequency and intensity laser modulation[J]. Applied Optics, 2003, 42(33): 6728-6738.

7. Bomse D S, Stanton A C, Silver J A. Frequency modulation and wavelength modulation spectroscopies: Comparison of experimental 
methods using a lead-salt diode laser[J]. Applied Optics, 1992, 31(6): 718-731.

8. Giusi G, Pace C, Crupi F. Cross-correlation-based trans-impedance amplifier for current noise measurements[J]. 2009, 37(6):781-792.

9. Kocsis L, Herman P, Eke A. The modified BeerLambert law revisited[J]. Physics in Medicine \& Biology, 2006, 51(5):N91.

10. Zhiqiang Song, Jiasheng Ni, Ying Shang, Chang Wang, Tongyu Liu, Yubin Wei. Study of longoptical-path white cell gas sensor with fiber coupling structure[J]. Journal of Optoelectronics. Laser, 2012, 23(6):1082-1085. 